\title{
Partitioning the temporal changes in abundance-based beta diversity into loss and gain components
}

\author{
Shinichi Tatsumi ${ }^{1,2, *}$, Ryosuke Iritani ${ }^{3}$, and Marc W. Cadotte ${ }^{1,4}$ \\ ${ }^{1}$ Department of Biological Sciences, University of Toronto Scarborough, Toronto, ON, Canada \\ ${ }^{2}$ Hokkaido Research Center, Forestry and Forest Products Research Institute, Hokkaido, Japan \\ ${ }^{3}$ RIKEN Interdisciplinary Theoretical and Mathematical Sciences (iTHEMS), Saitama, Japan \\ ${ }^{4}$ Department of Ecology and Evolutionary Biology, University of Toronto, Toronto, ON, Canada
}

*Corresponding author: Shinichi Tatsumi (community.ecologist@gmail.com)

\begin{abstract}
Spatial variation in species composition, or beta diversity, play central roles in gauging the structure of ecological communities. Here, we develop a new numerical method to partition the impacts of abundance losses and gains on the temporal changes in spatial beta diversity. We show that the temporal changes in pairwise dissimilarity measures (Ružička and Bray-Curtis indices) and a multiple-site measure (normalized Whittaker's beta) can be partitioned into six terms that reflect the losses and gains in species abundance. Our new method provides a unified approach to disentangle the temporal dynamics of spatial community structure by means of either incidenceor abundance-based data.
\end{abstract}




\section{INTRODUCTION}

2 Dissimilarity measures of species composition play central roles in gauging the spatial and 3 temporal structure of biodiversity (Wolda, 1981; Tamás, Podani, \& Csontos, 2001; Anderson et al.,

4 2011; Legendre \& Legendre, 2012; Chao \& Chiu, 2016). Among the properties that characterize

5 different measures of dissimilarity, one of the most significant is whether a given measure is 6 incidence-based (i.e., presence and absence of species) or abundance-based (Chao, Chazdon, \& 7 Shen, 2005; Legendre \& Legendre, 2012; Baselga, 2013). Incidence- and abundance-based 8 measures weigh rare and dominant species in different ways and thereby provide complementary 9 insights into community structure (Olsgard, Somerfield, \& Carr, 1997; Anderson et al., 2011;

10 Legendre \& Legendre, 2012; Li et al., 2016). Besides, abundance-based measures are relatively 11 robust against undersampling (Chao et al., 2005, 2014; Barwell, Isaac, \& Kunin, 2015) and are 12 capable of detecting changes in species dominance patterns even in the absence of richness 13 differences (Dornelas et al., 2014; Jones, Ripplinger, \& Collins, 2017). Extensions of existing 14 incidence-based methods to account for abundance can thus allow us to gain more comprehensive 15 pictures of spatiotemporal variation in species composition (Baselga, 2013; Chao et al., 2014).

Using incidence-based measures, previous studies have developed numerical methods to disentangle the species extinction and colonization processes that drive the compositional 18 dissimilarity across space, or beta diversity (Baeten et al., 2014; Rosenblad \& Sax, 2017; Li, 19 Bellard, Hu, \& Li, 2019; Tatsumi, Strengbom, Čugunovs, \& Kouki, 2020; Tatsumi, Iritani, \& Cadotte, 2021). Temporal decreases and increases in beta diversity (i.e., biotic homogenization and heterogenization, respectively) can both occur as a result of species extinctions or colonizations (i.e., changes from presence to absence, or vice versa) (McKinney \& Lockwood, 1999; Olden \& Poff, 2003; Socolar, Gilroy, Kunin, \& Edwards, 2016; Rosenblad \& Sax, 2017; Tatsumi et al., 2020). Specifically, extinctions lead to biotic homogenization when rare, infrequent species become regionally extinct, but otherwise result in heterogenization (Socolar et al., 2016; Rosenblad \& Sax, 2017; Tatsumi et al., 2020). Colonizations cause homogenization when new species become widespread or existing species increase their regional dominance, but drive heterogenization when new species colonize only a few sites (Socolar et al., 2016; Rosenblad \& Sax, 2017; Tatsumi et al., 2020). In our previous study (Tatsumi et al., 2021), we proposed a method to empirically partition such impacts of extinctions and colonizations on spatial beta diversity as quantified by incidencebased measures. 
Here, we develop a new method to partition the impacts of abundance losses and gains on spatial beta diversity by extending the previous incidence-based methods (Rosenblad \& Sax, 2017;

34 Li et al., 2019; Tatsumi et al., 2020, 2021). Similar to species extinctions and colonizations (i.e.,

35 binary changes between presence and absence), quantitative decreases and increases in local 36 abundance can drive either homogenization or heterogenization across space (Socolar et al., 2016).

37 The new method allows us to partition such temporal changes in spatial variation $\left(\Delta \beta=\beta^{\circ}-\beta\right.$,

38 where $\beta$ and $\beta^{\circ}$ are the values at $t=1$ and 2 , respectively) into distinct terms that reflect abundance

39 losses and gains, providing a unified approach for incidence and abundance data. Our method can

40 be applied to community-survey datasets (species $\times$ site matrices at $t=1$ and 2) with abundance

41 having been recorded in any form, including density, biomass, or the number of individuals, at

42 either absolute or relative scales.

\section{LOSS AND GAIN COMPONENTS OF ABUNDANCE-BASED BETA DIVERSITY}

45 Pairwise dissimilarity

46 We first describe the partitioning of temporal changes in pairwise dissimilarity measures, namely

47 Ružička ( $\left.\beta_{\text {Ruž}}\right)$ and Bray-Curtis indices $\left(\beta_{\mathrm{BC}}\right)$ (Bray \& Curtis, 1957; Ružička, 1958) (Fig. 1). These

48 measures are defined as $\beta_{\mathrm{Ruž}}=\left\{\sum_{i=1}^{S} u_{i}\right\} /\left\{\sum_{i=1}^{S}\left(c_{i}+u_{i}\right)\right\} \quad$ and $\quad \beta_{\mathrm{BC}}=$

$49\left\{\sum_{i=1}^{S} u_{i}\right\} /\left\{\sum_{i=1}^{S}\left(2 c_{i}+u_{i}\right)\right\}$, where $u_{i}$ is the part of abundance unique to either site, $c_{i}$ is the part of

50 abundance common to both sites, $i$ is species identity, and $S$ is the number of species. Here, let

$51 \beta_{p}=\left\{\sum_{i=1}^{S} u_{i}\right\} /\left\{\sum_{i=1}^{S}\left(p c_{i}+u_{i}\right)\right\}$, then $\beta_{\mathrm{Ružz}}=\beta_{1}$ and $\beta_{\mathrm{BC}}=\beta_{2}$.

52 Previous studies have defined six types of species extinctions and colonizations (i.e., changes

53 in presence-absence status) based on their impacts on spatial dissimilarity (Rosenblad \& Sax,

54 2017; Tatsumi et al., 2020, 2021). We here extend these definitions to account for abundance (Fig.

55 1). The first type is the reduction in $u_{i}$ (type 1 in Fig. 1); that is, for a given species $i$, a part of 56 abundance unique to either site $\left(u_{i}\right)$ at time $t=1$ becomes lost at time $t=2$. Type 2 is the case 57 where a part of abundance common to both sites $\left(c_{i}\right)$ becomes lost by equal amount in both sites.

58 Type 3 (or $3^{\prime}$ ) refers to the loss of $c_{i}$ in either site where the abundance is smaller than, or equal to, 59 that in the other site, whereby $c_{i}$ at $t=1$ turns into $u_{i}$ at $t=2$. Type 4 (or $4^{\prime}$ ) refers to the gain of $u_{i}$ 60 in the site where the abundance is larger than, or equal to, that in the other site. Type 5 is the case 61 where $c_{i}$ increases by equal amount in both sites. Type 6 refers to the gain of abundance in the site 62 where it was smaller than the other site, whereby $u_{i}$ turns into $c_{i}$. Types 1,5 , and 6 decrease $\beta_{p}$, 
(a) Pairwise dissimilarity measures
Ružička
Bray-Curtis
$\beta_{\mathrm{Ruž}}=\frac{\sum_{i=1}^{S} u_{i}}{\sum_{i=1}^{S}\left(c_{i}+u_{i}\right)}$
$\beta_{\mathrm{BC}}=\frac{\sum_{i=1}^{S} u_{i}}{\sum_{i=1}^{S}\left(2 c_{i}+u_{i}\right)}$
$i$ : Species identity
$u_{i}$ : Parts of abundance unique to either site
$c_{i}$ : Parts of abundance common to both sites

(b) Examples of losses and gains in species abundance

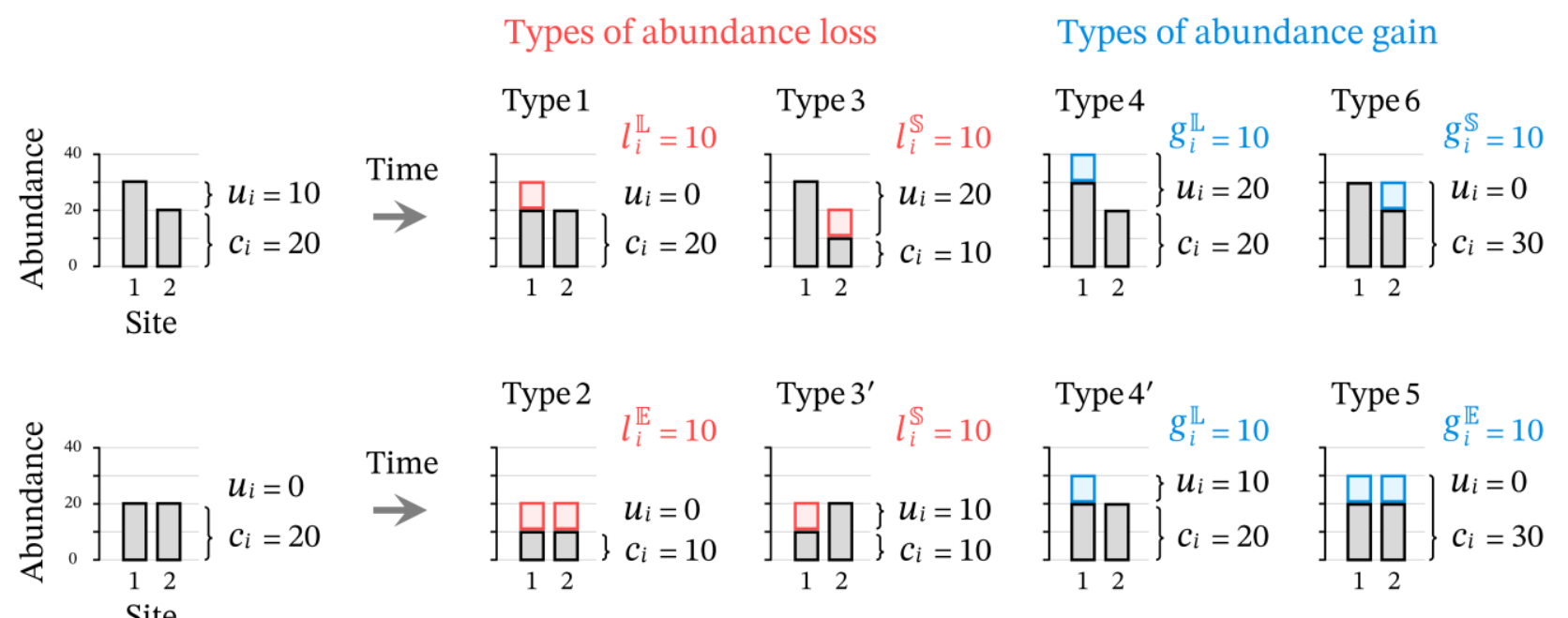

(c) Consequences of abundance losses and gains on dissimilarity

\begin{tabular}{|c|c|c|c|c|}
\hline \multirow[b]{2}{*}{ Type } & \multirow[b]{2}{*}{ Description } & \multicolumn{3}{|c|}{ Temporal changes in... } \\
\hline & & $u_{i}$ & $c_{i}$ & $\beta_{\text {Ruž and }} \beta_{B C}$ \\
\hline 1 & $\begin{array}{l}\text { Abundance }\left(a_{i}\right) \text { decreases in the site where } a_{i} \text { is larger }(\mathbb{L}) \text { than } \\
\text { that in the other site. }\end{array}$ & $-l_{i}^{\mathbb{L}}$ & 0 & - \\
\hline 2 & $a_{i}$ decreases by equal amount $(\mathbb{E})$ in both sites. & 0 & $-l_{i}^{\mathbb{E}}$ & + \\
\hline 3 or $3^{\prime}$ & $\begin{array}{l}a_{i} \text { decreases in the site where } a_{i} \text { is smaller }(\mathbb{S}) \text { than, or equal to, } \\
\text { that in the other site. }\end{array}$ & $+l_{i}^{\mathbb{S}}$ & $-l_{i}^{\mathbb{S}}$ & + \\
\hline 4 or $4^{\prime}$ & $\begin{array}{l}a_{i} \text { increases in the site where } a_{i} \text { is larger }(\mathbb{L}) \text { than, or equal to, } \\
\text { that in the other site. }\end{array}$ & $+g_{i}^{\mathbb{L}}$ & 0 & + \\
\hline 5 & $a_{i}$ increases by equal amount $(\mathbb{E})$ in both sites. & 0 & $+g_{i}^{\mathbb{E}}$ & - \\
\hline 6 & $\begin{array}{l}a_{i} \text { increases in the site where } a_{i} \text { is smaller }(\mathbb{S}) \text { than that in the } \\
\text { other site. }\end{array}$ & $-g_{i}^{\mathbb{S}}$ & $+g_{i}^{\mathbb{S}}$ & - \\
\hline
\end{tabular}

64 Figure 1. Schematic representation of the temporal changes in abundance-based beta 65 diversity and its loss and gain components. (a) Ružička and Bray-Curtis dissimilarity 66 indices, (b) examples of abundances losses and gains categorized into six types based on their impacts on dissimilarity, and (c) the description of the six types of abundances losses and gains. 
69 leading to homogenization, whereas types 2,3 , and 4 increase $\beta_{p}$, leading to heterogenization. We

70 denote the amount of changes in abundance that correspond to types $1,2,3,4,5$, and 6 as $l_{i}^{\mathbb{L}}, l_{i}^{\mathbb{E}}$,

$71 \quad l_{i}^{\mathbb{S}}, g_{i}^{\mathbb{L}}, g_{i}^{\mathbb{E}}$, and $g_{i}^{\mathbb{S}}$, respectively (Fig. 1).

72 It is possible for the abundance of a given species $i$ to change differently in the two sites

73 during the same time interval. Namely, if the abundance decreases in the site where it was larger

74 and increases in the other site where it was smaller, then beta diversity can potentially show no net

75 change. We refer to such offsetting replacements in species abundance as "hidden" dynamics

76 (Tatsumi et al. 2021). We denote the changes in abundance that fall under this definition as $d_{i}$. In

77 our partitioning method, $d_{i}$ is explicitly taken into account as a distinct form of abundance losses

78 and gains. In total, there are 24 possible combinations of abundance losses and gains, including the

79 hidden dynamics.

80 For compact notations, we denote the sum of a given variable across all species $(1,2, \ldots, S)$

81 using the uppercase letters (e.g., $\sum_{i}^{S} u_{i}=U$ and $\sum_{i}^{S} l_{i}^{\mathbb{L}}=L^{\mathbb{L}}$ ). To distinguish the variables at $t=1$

82 and $t=2$, we add the superscript ${ }^{\circ}$ to the latter (e.g., $\beta_{p}^{\circ}$ ). The temporal changes in $U$ and $C$ can

83 then be described as $\Delta U=U^{\circ}-U=-L^{\mathbb{L}}+L^{\mathbb{S}}+G^{\mathbb{L}}-G^{\mathbb{S}}$ and $\Delta C=C^{\circ}-C=-L^{\mathbb{E}}-L^{\mathbb{S}}+$

$84 G^{\mathbb{E}}+G^{\mathbb{S}}$ (Fig. 1). The relative changes in $U$ and $C$ can be defined as $\lambda_{U}=\Delta U / U$ and $\lambda_{C}=\Delta C / C$,

85 where $U$ and $C$ are both non-zero. We can additively partition the temporal changes in pairwise

86 dissimilarity $\left(\Delta \beta_{p}=\beta_{\mathrm{Ruž}}^{\circ}-\beta_{\mathrm{Ruž}}\right.$ when $p=1$ and $\Delta \beta_{p}=\beta_{\mathrm{BC}}^{\circ}-\beta_{\mathrm{BC}}$ when $\left.p=2\right)$ into six terms that

87 correspond to the six types of abundance changes:

$\Delta \beta_{p}=\beta_{p}^{\circ}-\beta_{p}$

$$
=\underbrace{-\frac{x}{U}\left(L^{\mathbb{L}}+h D\right)}_{\Delta \beta_{1}}+\underbrace{\frac{x}{C} L^{\mathbb{E}}}_{\Delta \beta_{2}}+\underbrace{\left(\frac{x}{C}+\frac{x}{U}\right)\left(L^{\mathbb{S}}+(1-h) D\right)}_{\Delta \beta_{3}}
$$

$$
+\underbrace{\frac{x}{U}\left(G^{\mathbb{L}}+h D\right)}_{\Delta \beta_{4}}+\underbrace{\frac{-x}{C} G^{\mathbb{E}}}_{\Delta \beta_{5}}+\underbrace{\left(-\frac{x}{C}-\frac{x}{U}\right)\left(G^{\mathbb{S}}+(1-h) D\right)}_{\Delta \beta_{6}}
$$

92 where $x=\frac{\beta_{p}\left(1-\beta_{p}\right)}{\left(1-\beta_{p}\right) \lambda_{C}+\beta_{p} \lambda_{U}+1}$ is a scaling factor and $h$ is a parameter associated with hidden 93 dynamics (see the second next paragraph for detail).

94 The first three terms and the last three terms in Eq. 1 represent the subtractive (abundance95 loss) components and the additive (abundance-gain) components of $\Delta \beta_{p}$. The first term is always 
96

97

negative, whereas the second and third terms are both positive. We therefore refer to the first term as subtractive homogenization $\left(\Delta \beta_{\text {Loss- }}\right)$ and the sum of second and third terms as subtractive heterogenization $\left(\Delta \beta_{\text {Loss }}\right.$ ). For similar reasons, we refer to the fourth term as additive heterogenization $\left(\Delta \beta_{\text {Gain+ }}\right)$ and the sum of fifth and sixth terms as additive homogenization $\left(\Delta \beta_{\text {Gain- }}\right)$. Depending on the ecological question at hand, one can sum the terms as $\Delta \beta_{\text {Loss }}=$ $\Delta \beta_{\text {Loss- }}+\Delta \beta_{\text {Loss }+}$ and $\Delta \beta_{\text {Gain }}=\Delta \beta_{\text {Gain }+}+\Delta \beta_{\text {Gain- }}$ to represent the total impacts of abundance losses and gains, respectively (Fig. 1).

The parameter $h$ in Eq. 1 ranges in the interval $[0,1]$ and determines the balance between two different ways the hidden dynamics can arise. First, the hidden dynamics can arise in such a way that the abundance first decreases in one site and then increases in the other site during a given time interval. That is, the abundance is lost in site 1 before it is gained in site 2 . Such abundance losses and gains correspond to the type-1 losses $\left(l_{i}^{\mathbb{L}}\right)$ and the type- $4^{\prime}$ gains $\left(g_{i}^{\mathbb{L}}\right)$, respectively. In Eq. 1 , the associated decreases and increases in $\beta_{p}$ are taken into account by adding $h d_{i}$ to $L^{\mathbb{L}}$ and $G^{\mathbb{L}}$ in the first and fourth terms, respectively. The second form of hidden dynamics is where the abundance is gained prior to getting lost. Such gains and losses correspond to the type-5 gains $\left(g_{i}^{\mathbb{S}}\right)$ and the type- $2^{\prime}$ losses $\left(l_{i}^{\mathbb{S}}\right)$, respectively. The resultant decreases and increases in $\beta_{p}$ are reflected in Eq. 1 by adding $(1-h) d_{i}$ to $G^{\mathbb{S}}$ and $L^{\mathbb{S}}$ in the sixth and third terms, respectively. As such, the parameter $h$ determines how the order of abundance losses and gains affect $\Delta \beta_{p}$ via $d_{i}$. When $h=$ 0 , abundance is always lost before it is gained within a given time interval, and vice versa when $h$ $=1$. When $h=0.5$, neutrality is assumed with regards to the order of abundance losses and gains.

\section{Multiple-site variation}

Our partitioning approach can not only be applied to pairwise measures but also to multiple-site dissimilarity measures. We here consider the normalized Whittaker's beta (Harrison, Ross, \& Lawton, 1992) defined as $(\gamma / \bar{\alpha}-1) /(N-1)$, where $\gamma$ is the regional species richness, $\bar{\alpha}$ is the mean local species richness, and $N$ is the number of sites. While the original Whittaker's beta $(\gamma / \bar{\alpha})$ ranges in the interval $[1, N]$ (Whittaker, 1960), the normalized measure ranges in the interval $[0,1]$ (Harrison et al., 1992). This normalized measure has been extended by Baselga (2017) to account for abundance. We denote the extended measure as $\beta_{\mathrm{HB}}$ (Harrison-Baselga's beta) defined as: 
$\beta_{\mathrm{HB}}=\frac{N \cdot \sum_{i}^{S} \max _{j}\left\{a_{i j}\right\}-A}{(N-1) A}$

where $a_{i j}$ is the abundance of species $i$ in site $j, N$ is the number of sites, $S$ is the number of species, $\max _{j}\left\{a_{i j}\right\}$ is the maximum abundance of species $i$ across all sites, and $A$ is the total abundance of

129 all species in all sites (i.e., $A=\sum_{i}^{S} \sum_{j}^{N} a_{i j}$ ). For notational simplicity, we denote $\max _{j}\left\{a_{i j}\right\}$ as $m_{i}$ 130 and $\sum_{i}^{S} \max _{j}\left\{a_{i j}\right\}$ as $M$. We also denote the abundance of species $i$ summed across the sites $\left(\sum_{j}^{N} a_{i j}\right)$ 131 as $a_{i+}$.

132 The variables $l_{i}^{\mathbb{L}}, l_{i}^{\mathbb{E}}, l_{i}^{\mathbb{S}}, g_{i}^{\mathbb{L}}, g_{i}^{\mathbb{E}}$, and $g_{i}^{\mathbb{S}}$, which we defined above to describe the changes in $u_{i}$ 133 and $c_{i}$ (Fig. 1), can be extended to represent the changes in the maximum abundance $\left(m_{i}\right)$ and the 134 total abundance $\left(a_{i+}\right)$ of species $i$. First, we extend the definition of $l_{i}^{\mathbb{L}}$, such that it denotes the 135 temporal decreases in abundance associated with both $m_{i}$ and $a_{i+}$. This type of abundance loss leads 136 to reductions in $m_{i}$ by the amount equal to $l_{i}^{\mathbb{L}}$. For example, $m_{i}$ decreases by 10 when $l_{i}^{\mathbb{L}}=10$. The 137 amount of reduction in $a_{i+}$, on the other hand, depends on the number of sites in which a given 138 species $i$ showed its maximum abundance at $t=1$. This dependency can be taken explicitly into 139 account by adding the subscript $n$ to $l_{i}^{\mathbb{L}}$, such that $l_{i n}^{\mathbb{L}}$ denotes the decreases in the maximum 140 abundance across $n$ sites. Such abundance losses cause reductions in $a_{i+}$ by the amount equal to $141 n l_{i n}^{\mathbb{L}}$. For instance, $a_{i+}$ decreases by 10 and 20 when $l_{i 1}^{\mathbb{L}}=10$ and $l_{i 2}^{\mathbb{L}}=10$, respectively, whereas $142 m_{i}$ decreases by 10 in either cases. Similarly, we define $g_{i n}^{\mathbb{L}}$ to denote the increases in the maximum 143 abundance of species $i$ across $n$ sites.

144 The extended definition of $l_{i}^{\mathbb{E}}$ represents the abundance losses in all sites $(N)$ across which a 145 given specie $i$ showed the same abundance at $t=1$. In this case, $m_{i}$ and $a_{i+}$ decrease by $l_{i}^{\mathbb{E}}$ and $N l_{i}^{\mathbb{E}}$, 146 respectively. Thus, $l_{i}^{\mathbb{E}}$ can be seen as a special case of $l_{i n}^{\mathbb{L}}$ when $n=N$. We therefore rewrite $l_{i}^{\mathbb{E}}$ as $147 l_{i N}^{\mathbb{L}}$ hereafter. Similarly, $g_{i}^{\mathbb{E}}$ is rewritten as $g_{i N}^{\mathbb{L}}$ to indicate the gains in abundance across all sites 148 that result in a given species $i$ having the same abundance at $t=2$.

149 The extended definition of $l_{i}^{\mathbb{S}}$ refers to the abundance losses associated with decreases in $a_{i+}$ 150 but not $m_{i}$. We use the subscript $n$ to let $l_{\text {in }}^{\mathbb{S}}$ denote the abundance losses in $n$ sites across which a 151 given species $i$ showed smaller abundance than, or equal abundance to, $m_{i}$ at both $t=1$ and $t=2$.

152 This type of abundance losses lead to a reduction in $a_{i+}$ by $n l_{i n}^{\mathbb{S}}$ but do not alter $m_{i}$. Similarly, we 
153 define $g_{i n}^{\mathbb{S}}$ as the increase in abundance across $n$ sites where species $i$ showed smaller abundance than, or equal abundance to, $m_{i}$ at both $t=1$ and $t=2$.

Hidden dynamics can not only occur in cases with two sites $(N=2)$ but also with multiple sites $(N>2)$. As such, we extend the definition of hidden dynamics to refer to the abundance losses in site(s) where a given species $i$ showed its maximum abundance at $t=1$ and the offsetting gains in the other site(s). By adding subscripts $p$ and $q$ to $d_{i}$, we write $d_{i p q}$ to denote the abundance losses in $p$-number of sites and gains in $q$-number of sites $(p+q \leq N)$ occurring within the same time interval.

We denote the variables $l_{i n}^{\mathbb{L}}, l_{i n}^{\mathbb{S}} g_{i n}^{\mathbb{L}}, g_{i n}^{\mathbb{S}}$, and $d_{i p q}$ summed across all species $(i=1,2, \ldots, S)$ as $L_{n}^{\mathbb{L}}, L_{n}^{\mathbb{S}}, G_{n}^{\mathbb{L}}, G_{n}^{\mathbb{S}}$, and $D_{p q}$, respectively. Equation 1 can be rewritten using the extended definitions by replacing $L^{\mathbb{L}}, L^{\mathbb{E}}, L^{\mathbb{S}}, G^{\mathbb{L}}, G^{\mathbb{E}}, G^{\mathbb{S}}$, and $D$ with $L_{1}^{\mathbb{L}}, L_{2}^{\mathbb{L}}, L_{1}^{\mathbb{S}}, G_{1}^{\mathbb{L}}, G_{2}^{\mathbb{L}}, G_{1}^{\mathbb{S}}$, and $D_{11}$, respectively.

To partition $\Delta \beta_{\mathrm{HB}}$, we first consider the case of two communities $(N=2)$. The temporal changes in $M$ and $A$ can be described as $\Delta M=M^{\circ}-M=-L_{1}^{\mathbb{L}}-L_{2}^{\mathbb{L}}+G_{1}^{\mathbb{L}}+G_{2}^{\mathbb{L}}$ and $\Delta A=A^{\circ}-$ $A=-L_{1}^{\mathbb{L}}-2 L_{2}^{\mathbb{L}}-L_{1}^{\mathbb{S}}+G_{1}^{\mathbb{L}}+2 G_{2}^{\mathbb{L}}+G_{1}^{\mathbb{S}}$. The relative changes in $M$ and $A$ can be defined as $\lambda_{M}=$

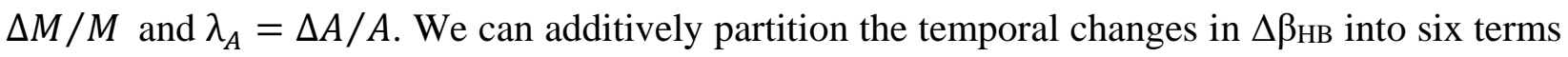
that correspond to the six types of abundance changes:

$$
\begin{gathered}
=\underbrace{\left(\frac{-y}{M}+\frac{y}{A}\right)\left(L_{1}^{\mathbb{L}}+h D_{11}\right)}_{\Delta \beta_{1}}+\underbrace{\left(\frac{-y}{M}+\frac{2 y}{A}\right) L_{2}^{\mathbb{L}}}_{\Delta \beta_{2}}+\underbrace{\frac{y}{A}\left(L_{1}^{\mathbb{S}}+(1-h) D_{11}\right)}_{\Delta \beta_{3}} \\
+\underbrace{\left(\frac{y}{M}-\frac{y}{A}\right)\left(G_{1}^{\mathbb{L}}+h D_{11}\right)}_{\Delta \beta_{4}}+\underbrace{\left(\frac{y}{M}-\frac{2 y}{A}\right) G_{2}^{\mathbb{L}}}_{\Delta \beta_{5}}+\underbrace{\frac{-y}{A}\left(G_{1}^{\mathbb{S}}+(1-h) D_{11}\right)}_{\Delta \beta_{6}}
\end{gathered}
$$

where $y=\frac{N M}{(N-1)\left(\lambda_{A}+1\right) A}$ is a scaling factor. We define the six terms in Eq. 3 as the loss and gain components of temporal changes in multiple-site dissimilarity $\left(\beta_{\mathrm{HB}}\right)$ at $N=2$. The definitions of these components are in line with those based on the Ružička $\left(\beta_{\text {Ruž }}\right)$ Bray-Curtis indices $\left(\beta_{\mathrm{BC}}\right)(\mathrm{Eq}$. $1)$. generalized as: 
$\Delta \beta_{\mathrm{HB}}=\beta_{\mathrm{HB}}^{\circ}-\beta_{\mathrm{HB}}$

181

$$
=\underbrace{\sum_{n=1}^{N}\left(\frac{-y}{M}+\frac{n y}{A}\right) L_{n}^{\mathbb{L}}+\sum_{\{\mid p+q \leq N\}}\left(\frac{-y}{M}+\frac{p y}{A}\right) h D_{p q}}_{\left\{\beta_{\text {Loss- }} \text { or } \Delta \beta_{\text {Loss }}\right.}
$$

$$
+\underbrace{\sum_{n}^{N-1} \frac{n y}{A} L_{n}^{\mathbb{S}}+\sum_{\{p, q \mid p+q \leq N\}} \frac{p y}{A}(1-h) D_{p q}}_{\Delta \beta_{\text {Loss }}}
$$

$$
+\underbrace{\sum_{j=1}^{N}\left(\frac{y}{M}-\frac{n y}{A}\right) G_{n}^{\mathbb{L}}+\sum_{\text {Gain- }} \text { or } \Delta \beta_{\text {Gain }+}\left(\frac{y}{M}-\frac{q y}{A}\right) h D_{p q}}_{\{p, q \mid p+q \leq N\}}
$$

$$
+\underbrace{\sum_{n}^{N-1} \frac{-n y}{A} G_{n}^{\mathbb{S}}+\sum_{\{p, q \mid p+q \leq N\}} \frac{-q y}{A}(1-h) D_{p q}}_{\Delta \beta_{\text {Gain- }}}
$$

\section{DISCUSSION}

188 Here, we developed a new method that allows us to partition the temporal changes in beta diversity into distinct terms that reflect abundance losses and gains. The method provides a unified approach to disentangle the dynamics of spatial community structure by means of either incidence or abundance data.

\section{ACKNOWLEDGEMENTS}

194 ST was supported by a JSPS Overseas Research Fellowship (No. 201860500) from the Japan 195 Society for the Promotion of Science (JSPS). RI was supported by JSPS grants (No. 19K22457, 19K23768, and 20K15882).

\section{REFERENCES} the practicing ecologist. Ecology Letters, 14(1), 19-28. doi:10.1111/j.1461- 
Baeten, L., Warton, D. I., Van Calster, H., De Frenne, P., Verstraeten, G., Bonte, D., ... Verheyen, K. (2014). A model-based approach to studying changes in compositional heterogeneity. Methods in Ecology and Evolution, 5(2), 156-164. doi:10.1111/2041-210X.12137

Barwell, L. J., Isaac, N. J. B., \& Kunin, W. E. (2015). Measuring $\beta$-diversity with species abundance data. Journal of Animal Ecology, 84(4), 1112-1122. doi:10.1111/13652656.12362

Baselga, A. (2013). Separating the two components of abundance-based dissimilarity: Balanced changes in abundance vs. abundance gradients. Methods in Ecology and Evolution, 4(6), 552557. doi:10.1111/2041-210X.12029

Baselga, A. (2017). Partitioning abundance-based multiple-site dissimilarity into components:

Bray, J. R., \& Curtis, J. T. (1957). An ordination of the upland forest communities of southern Wisconsin. Ecological Monographs, 27(4), 325-349. doi:10.2307/1942268

Chao, A., Chazdon, R. L., \& Shen, T. J. (2005). A new statistical approach for assessing similarity doi:10.1111/j.1461-0248.2004.00707.x

Chao, A., \& Chiu, C. H. (2016). Bridging the variance and diversity decomposition approaches to beta diversity via similarity and differentiation measures. Methods in Ecology and Evolution, 7(8), 919-928. doi:10.1111/2041-210X.12551

Chao, A., Gotelli, N. J., Hsieh, T. C., Sander, E. L., Ma, K. H., Colwell, R. K., \& Ellison, A. M. (2014). Rarefaction and extrapolation with Hill numbers: A framework for sampling and estimation in species diversity studies. Ecological Monographs, 84(1), 45-67. doi:10.1890/13-0133.1

Dornelas, M., Gotelli, N. J., McGill, B., Shimadzu, H., Moyes, F., Sievers, C., \& Magurran, A. E. (2014). Assemblage time series reveal biodiversity change but not systematic loss. Science, 344(6181), 296-299. doi:10.1126/science.1248484

Harrison, S., Ross, S. J., \& Lawton, J. H. (1992). Beta diversity on geographic gradients in Britain. Journal of Animal Ecology, 61(1), 151. doi:10.2307/5518

Jones, S. K., Ripplinger, J., \& Collins, S. L. (2017). Species reordering, not changes in richness, drives long-term dynamics in grassland communities. Ecology Letters, 20(12), 1556-1565. 
doi:10.1111/ele.12864

Legendre, P., \& Legendre, L. (2012). Numerical Ecology. Elsevier.

236 Li, S., Cadotte, M. W., Meiners, S. J., Pu, Z., Fukami, T., \& Jiang, L. (2016). Convergence and

237 divergence in a long-term old-field succession: The importance of spatial scale and species abundance. Ecology Letters, 19(9), 1101-1109. doi:10.1111/ele.12647

Li, X., Bellard, C., Hu, F., \& Li, H. (2019). A comprehensive formula for decomposing change in community similarity into introduction and extinction events. Ecography, 42(10), 1714-1716. doi:10.1111/ecog.04613

McKinney, M. L., \& Lockwood, J. L. (1999). Biotic homogenization: A few winners replacing many losers in the next mass extinction. Trends in Ecology \& Evolution, 14(11), 450-453. doi:10.1016/S0169-5347(99)01679-1

Olden, J. D., \& Poff, N. L. (2003). Toward a mechanistic understanding and prediction of biotic homogenization. The American Naturalist, 162(4), 442-460. doi:10.1086/378212

Olsgard, F., Somerfield, P., \& Carr, M. (1997). Relationships between taxonomic resolution and data transformations in analyses of a macrobenthic community along an established pollution gradient. Marine Ecology Progress Series, 149(5), 173-181. doi:10.3354/meps149173

Rosenblad, K. C., \& Sax, D. F. (2017). A new framework for investigating biotic homogenization and exploring future trajectories: Oceanic island plant and bird assemblages as a case study. Ecography, 40(9), 1040-1049. doi:10.1111/ecog.02652

Ružička, M. (1958). Anwendung mathematisch-statisticher Methoden in der Geobotanik (synthetische Bearbeitung von Aufnahmen). Biológia, Bratislava, 13, 647-661.

Socolar, J. B., Gilroy, J. J., Kunin, W. E., \& Edwards, D. P. (2016). How should beta-diversity inform biodiversity conservation? Trends in Ecology \& Evolution, 31(1), 67-80. doi:10.1016/j.tree.2015.11.005

Tamás, J., Podani, J., \& Csontos, P. (2001). An extension of presence/absence coefficients to abundance data: A new look at absence. Journal of Vegetation Science, 12(3), 401-410. doi: $10.2307 / 3236854$

Tatsumi, S., Iritani, R., \& Cadotte, M. W. (2021). Temporal changes in spatial variation: partitioning the extinction and colonisation components of beta diversity. Ecology Letters, 24(5), 1063-1072. doi:10.1111/ele.13720

Tatsumi, S., Strengbom, J., Čugunovs, M., \& Kouki, J. (2020). Partitioning the colonization and 
extinction components of beta diversity across disturbance gradients. Ecology, 101(12), e03183. doi:10.1002/ecy.3183

267 Whittaker, R. H. (1960). Vegetation of the Siskiyou Mountains, Oregon and California. Ecological

268 Monographs, 30(3), 279-338. doi:10.2307/1943563

269 Wolda, H. (1981). Similarity indices, sample size and diversity. Oecologia, 50(3), $296-302$. 